BMJ Open

Diabetes

Research

\& Care

\section{Increased plasma neurofilament light chain levels in patients with type-1 diabetes with impaired awareness of hypoglycemia}

To cite: Sampedro F, Stantonyonge N, Martínez-Horta S, et al. Increased plasma neurofilament light chain levels in patients with type1 diabetes with impaired awareness of hypoglycemia. BMJ Open Diab Res Care 2020;8:e001516. doi:10.1136/ bmjdrc-2020-001516

FS and NS contributed equally.

Received 4 May 2020 Revised 23 May 2020 Accepted 30 May 2020

Check for updates

(C) Author(s) (or their employer(s)) 2020. Re-use permitted under CC BY-NC. No commercial re-use. See rights and permissions. Published by BMJ.

${ }^{1}$ Neurology, Hospital de la Santa Creu i Sant Pau, Barcelona, Spain

${ }^{2}$ Endocrinology, Hospital de la Santa Creu i Sant Pau, Barcelona, Spain ${ }^{3}$ School of Medicine, Autonomous University of Barcelona, Barcelona, Catalunya, Spain ${ }^{4}$ Neuroradiology, Hospital de la Santa Creu i Sant Pau, Barcelona, Spain

Correspondence to Dr Ana Chico; achicob@santpau.cat

\section{ABSTRACT}

Introduction Impaired awareness of hypoglycemia (IAH) is a common complication in patients with type- 1 diabetes (T1D). IAH is a major risk factor for severe hypoglycemic events, leading to adverse clinical consequences and cerebral damage. Non-invasive, cost-effective, and logistically efficient biomarkers for this condition have not been validated. Here, we propose plasma neurofilament light chain (NfL) levels as a biomarker of neuroaxonal damage in patients with T1D-IAH.

Research design and methods 54 patients were included into the study (18 T1D-IAH, 18 T1D with normal awareness of hypoglycemia (NAH) and 18 healthy controls). We measured plasma NfL levels and studied cerebral gray matter alterations on MRI.

Results We found that NfL levels were increased in patients with T1D-IAH compared with patients with T1D$\mathrm{NAH}$ and healthy controls. Importantly, increased NfL levels correlated with reduced cerebral gray matter volume and increased IAH severity in patients with T1D-IAH.

Conclusion Overall, our findings identify plasma NfL levels as a potential biomarker of cerebral damage in this population, motivating further confirmatory studies with potential implications in clinical trials.

\section{INTRODUCTION}

About one in five patients with type 1 diabetes (T1D) have impaired awareness of hypoglycemia (IAH). ${ }^{1}$ IAH represents a risk factor for severe and recurrent hypoglycemic events, which can lead to brain damage. ${ }^{23}$ As no effective treatment has been clearly established, ${ }^{4}$ biomarkers capable of detecting and quantifying cerebral damage in this population are needed for the design of novel preventive or therapeutic strategies.

On the one hand, cerebral atrophy or functional impairment can be assessed using neuroimaging techniques. In fact, a structural and functional brain compromise has been described in patients with T1D-IAH. ${ }^{5}$ However, neuroimaging techniques have important limitations in terms of specificity,

\section{Significance of this study}

What is already known about this subject?

- Patients with type 1 diabetes (T1D) with impaired awareness of hypoglycemia $(\mathrm{IAH})$ are in higher risk of severe hypoglycemia (SH).

- $\mathrm{SH}$ is associated with adverse clinical consequences and cerebral damage.

- There is no validated biomarker for this subgroup of patients.

What are the new findings?

- Plasma neurofilament light chain (NfL) levels were increased in patients with T1D-IAH compared to patients with T1D with normal awareness of hypoglycemia and healthy controls.

- Increased NfL levels correlated with reduced cerebral gray matter volume and increased IAH severity in patients with T1D-IAH.

How might these results change the focus of research or clinical practice?

- Plasma Nfl levels could be used as a non-invasive, cost-effective, and logistically efficient biomarker of neuroaxonal damage in patients with T1D-IAH.

logistics, standardization, scalability, and cost-efficiency. On the other hand, while cerebrospinal fluid biomarkers of cerebral damage overcome some of these limitations, they require an invasive lumbar puncture procedure.

In recent years, research has focused on the detection of blood biomarkers of neural damage. Clearly, cost-efficient blood biomarkers would represent an accessible and centralizable alternative. However, the main challenge for the development of an adequate technology in this context has been the low blood concentration of proteins derived from the central nervous system (CNS). Importantly, in the last few years, the 
Single Molecule Array (Simoa) technology has shown unprecedented precision and sensitivity (ie, femtomolar range) for the quantification of blood biomarkers of brain damage. ${ }^{6}$ In particular, increased levels of neurofilament light chain (NfL) in blood have shown a great potential for the detection of neurodegeneration (neuronal loss and axonal damage), traumatic brain injury, and cerebrovascular accidents. ${ }^{7}$

In this brief report, we investigate whether patients with T1D with hypoglycemia unawareness show increased levels of plasma NfL compared with patients with normal awareness of hypoglycemia (NAH) and healthy controls (HC). Additionally, we explore whether abnormally increased NfL levels in this population correlate with IAH severity and reduced cerebral gray matter volume (GMV).

\section{MATERIALS AND METHODS}

\section{Sample and assessments}

A total of 54 participants were included in this study: 36 patients with T1D and 18 HC. Eighteen patients with T1D fulfilled clinical criteria for IAH (T1D-IAH) and the other 18 patients had NAH (T1D-NAH). Both T1D groups were selected as to have similar sociodemographics and T1D duration. The Clarke score was used for the IAH classification, which is based on eight questions that allow the determination of the glycemic threshold in which the patients notice the hypoglycemic symptoms. ${ }^{8}$ A Clarke score $\geq 4$ was used to identify patients with T1DIAH, whereas patients with T1D-NAH showed a score $<3$. The Clarke method comprises the following set of questions designed to characterize the participant's exposure to hypoglycemia: Do you feel hypoglycemia symptoms when your blood glucose is low?, Have you lost symptoms of hypoglycemia that used to occur when your blood glucose is low?, Check all your symptoms of hypoglycemia over the last 6 months, If your answered 'yes' to any of the above question, how often do you have it?, Check all your symptoms of hypoglycemia over the last 12 months. If your answered 'yes' to any of the above question, how often do you have it? How often did you have an episode of hypoglycemia with symptoms during the last month? How often did you have an episode of hypoglycemia without symptoms during the last month? What was the lowest blood glucose level before feeling the symptoms of hypoglycemia? How often did you have symptoms of hypoglycemia when your blood glucose level was low?

The following exclusion criteria were applied: previous clinical diagnosis of cognitive impairment or neurological disorders, use of medications affecting the CNS, and MRI contraindications or intolerance. None of the participants in this study had any previous medical condition (apart from T1D) known to significantly alter blood NfL levels or cerebral GMV.

Both T1D groups were under similar insulin treatment, either as basal-bolus regimen or continuous subcutaneous insulin infusion (with short-acting insulin analogs). Sociodemographic, clinical, and laboratory data (diabetes and cardiovascular indicators) were recorded for each patient. Quantification of IAH severity was assessed, on one hand, by Clarke scores. On the other hand, we also recorded the number of severe hypoglycemic episodes during the last 5 years and the number of moderate hypoglycemic events during the last month. A severe hypoglycemic episode associated loss of consciousness, confusion/disorientation, seizures, or coma and required the assistance of a third person. A moderate hypoglycemic event was defined as any non-severe episode requiring the use of measures with impact on the daily life (interrupting work routine, stopping the car while driving, and so on). We combined both indicators to obtain a single indicator of the number of severe or moderate hypoglycemic episodes, providing a representative indicator of the clinical consequences of IAH.

\section{Plasma NfL procedure}

Blood was collected in EDTA tubes, centrifuged at $2000 \mathrm{~g}$ for $10 \mathrm{~min}$ and the plasma aliquoted in polypropylene tubes, and frozen at $-80^{\circ} \mathrm{C}$ according to international consensus recommendations until analysis. Plasma NfL levels were measured with the Simoa Human NF-light Advantage kit using the Single Molecule Array technology (Simoa; Quanterix) ${ }^{9}$ in a SR-X Biomarker detection system by following the manufacturer's instructions.

\section{Neuroimaging acquisition and preprocessing}

T1-weighted MRI scans were acquired in a Philips 3-Tesla station using a magnetization-prepared rapid acquisition with gradient echo protocol using the following parameters: flip angle $8^{\circ}$, repetition time $6.7 \mathrm{~s}$, echo time 3.19 $\mathrm{ms}$, pixel bandwidth 241, slice thickness $1.2 \mathrm{~mm}$, and an acquisition matrix of size $256 \times 256$. All patients had available T1-MRI imaging, but nine subjects from the HC group did not have MRI data.

GMVs of the frontal, temporal, parietal, and occipital lobes for each participant were computed using the FreeSurfer 6.0 software package. Details about the standard FreeSurfer segmentation pipeline are available elsewhere (https://surfer.nmr.mgh.harvard.edu/). All preprocessed images were visually inspected to confirm the absence of segmentation errors.

\section{Statistical analyses}

Clinical, sociodemographics, NfL levels, and imaging data were compared across groups using one-way ANOVA (except $\chi^{2}$ for sex differences). Pairwise group differences were examined by general linear models including age, sex, education, and T1D duration as covariates of no interest. A logistic regression was also performed. In addition, we explored whether increased plasma NfL levels correlated with increased IAH severity or reduced GMV in patients with T1D. For that, we used one-sided Pearson's correlation coefficients using the same nuisance covariates, for which a $\mathrm{p}<0.05$ was considered significant. 
Table 1 Clinical, demographic, biomarker, and imaging information across groups

\begin{tabular}{|c|c|c|c|c|}
\hline & T1D-IAH & T1D-NAH & $\mathrm{HC}$ & $\begin{array}{l}\text { Significance } \\
\text { ( } p \text { value) }\end{array}$ \\
\hline $\mathrm{n}$ & 18 & 18 & 18 & \\
\hline Age (years) & $55.5 \pm 13.5$ & $57.4 \pm 8.1$ & $54.7 \pm 13.7$ & 0.79 \\
\hline Sex (\% female) & $61 \%$ & $27 \%$ & $44 \%$ & 0.13 \\
\hline Education (years) & $14.8 \pm 5.1$ & $14.7 \pm 4.4$ & $13.6 \pm 3.5$ & 0.64 \\
\hline Plasma NfL (pg/mL) & $18.22 \pm 11.7$ & $13.27 \pm 6.7$ & $8.15 \pm 4.6$ & $0.002^{*}$ \\
\hline T1D duration & $31.7 \pm 12.8$ & $30.2 \pm 8.6$ & NA & 0.66 \\
\hline $\mathrm{HbA1c}(\%)$ & $7.57 \pm 1.1$ & $7.60 \pm 0.8$ & NA & 0.93 \\
\hline Body mass index $\left(\mathrm{kg} / \mathrm{m}^{2}\right)$ & $25.9 \pm 6.6$ & $27.2 \pm 3.8$ & NA & 0.49 \\
\hline Mean arterial pressure $(\mathrm{mm} \mathrm{Hg})$ & $93.6 \pm 15.6$ & $101.8 \pm 13.6$ & NA & 0.10 \\
\hline LDL-cholesterol (mg/dL) & $96.3 \pm 27.4$ & $94.6 \pm 21.3$ & NA & 0.84 \\
\hline HDL-cholesterol (mg/dL) & $71.4 \pm 16.5$ & $63.7 \pm 19.1$ & NA & 0.21 \\
\hline Clarke score & $4.7 \pm 0.9$ & $1.0 \pm 0.7$ & NA & $<0.001^{*}$ \\
\hline Number of severe or moderate hypoglycemic episodes & $4.89 \pm 6.3$ & $0.61 \pm 1.0$ & NA & $0.007^{\star}$ \\
\hline Minimental state examination & $29.56 \pm 0.6$ & $29.61 \pm 0.8$ & NA & 0.81 \\
\hline Frontal lobe GMV $\left(\mathrm{mm}^{3} \times 10^{-3}\right)$ & $138.6 \pm 9.9$ & $150.3 \pm 13.5$ & $165.0 \pm 17.6$ & $<0.001^{*}$ \\
\hline Temporal lobe GMV $\left(\mathrm{mm}^{3} \times 10^{-3}\right)$ & $93.0 \pm 7.2$ & $102.3 \pm 9.7$ & $100.8 \pm 9.6$ & $0.007^{*}$ \\
\hline Parietal lobe GMV $\left(\mathrm{mm}^{3} \times 10^{-3}\right)$ & $89.6 \pm 8.7$ & $97.3 \pm 9.4$ & $108.2 \pm 8.8$ & $<0.001^{*}$ \\
\hline Occipital lobe GMV $\left(\mathrm{mm}^{3} \times 10^{-3}\right)$ & $41.7 \pm 4.8$ & $48.3 \pm 6.8$ & $45.1 \pm 6.6$ & $0.008^{*}$ \\
\hline
\end{tabular}

Values are expressed as mean \pm SD or percentage.

${ }^{*} \mathrm{P}<0.05$ three-group ANOVA $\chi^{2}$ or t-test between T1D groups.

ANOVA, analysis of variance; GMV, gray matter volumes; $\mathrm{HC}$, healthy controls; HDL, high-density lipoprotein; IAH, impaired awareness of hypoglycemia; LDL, low-density lipoprotein; NA, not applicable or available; NAH, normal awareness of hypoglycemia; NfL, neurofilament light chain; T1D, type-1 diabetes.

\section{RESULTS}

Table 1 shows the sample characteristics of this study. All groups had similar sociodemographics. There were no significant differences between T1D groups in terms of disease duration, cognitive status, or cardiovascular risk factors. However, as expected, patients with T1D-IAH showed increased Clarke scores and number of severe or moderate hypoglycemic events in the last month, reflecting common clinical consequences of IAH.

Significant differences were found across groups in plasma NfL, progressively increasing from HC to T1DNAH and T1D-IAH. Pairwise NfL differences between T1D-IAH and T1D-NAH were significant after controlling for age, sex, education, and disease duration $(\beta=0.039$, $\mathrm{p}=0.038$ ). A logistic regression to classify patients with T1D-IAH versus T1-NAH including all factors listed in table 1 (except for Clarke scores and number of hypoglycemic episodes) yielded the following results. Significance was only achieved by NfL levels $(p=0.038)$, but the following close-to-significance factors were also observed: frontal GMV ( $\mathrm{p}=0.062)$, temporal GMV $(\mathrm{p}=0.059)$, age $(\mathrm{p}=0.066)$, sex $(\mathrm{p}=0.064)$, and body mass index $(\mathrm{p}=0.054)$. Similarly, progressively decreasing GMV was observed across groups and cerebral lobules, and pairwise differences between T1D-IAH and T1D-NAH remained significant after controlling for the same nuisance covariates.
Finally, the following exploratory associations between increased NfL levels in patients with T1D and IAH severity and reduced cerebral GMV were observed. Within the T1D-IAH group, increased NfL levels correlated with lower frontal GMV $(\mathrm{r}=-0.51, \mathrm{p}=0.030)$ and increased number of severe or moderate hypoglycemic events $(\mathrm{r}=0.49, \mathrm{p}=0.038)$. When considering all patients with T1D, NfL levels correlated with Clarke scores $(\mathrm{r}=0.34$, $\mathrm{p}=0.029)$ and the number of severe or moderate hypoglycemic events $(\mathrm{r}=0.45, \mathrm{p}=0.005)$. Finally, although non-significant, NfL levels showed a mild association with temporal and parietal GMV ( $\mathrm{r}=-0.28, \mathrm{p}=0.051$ and $\mathrm{r}=-0.21, \mathrm{p}=0.11$, respectively).

Figure 1 displays NfL differences across groups and a subset of these associations, using in some cases $\log \mathrm{NfL}$ values to better illustrate group differences or correlations, for which all statistical results remained significant.

\section{DISCUSSION}

Our results reinforce the presence of increased brain damage in patients with T1D-IAH. Plasma NfL levels were increased in this population and correlated with reduced cortical gray matter and IAH severity. Therefore, plasma NfL could be a promising cost-efficient biomarker of neural damage in patients with T1D-IAH. 

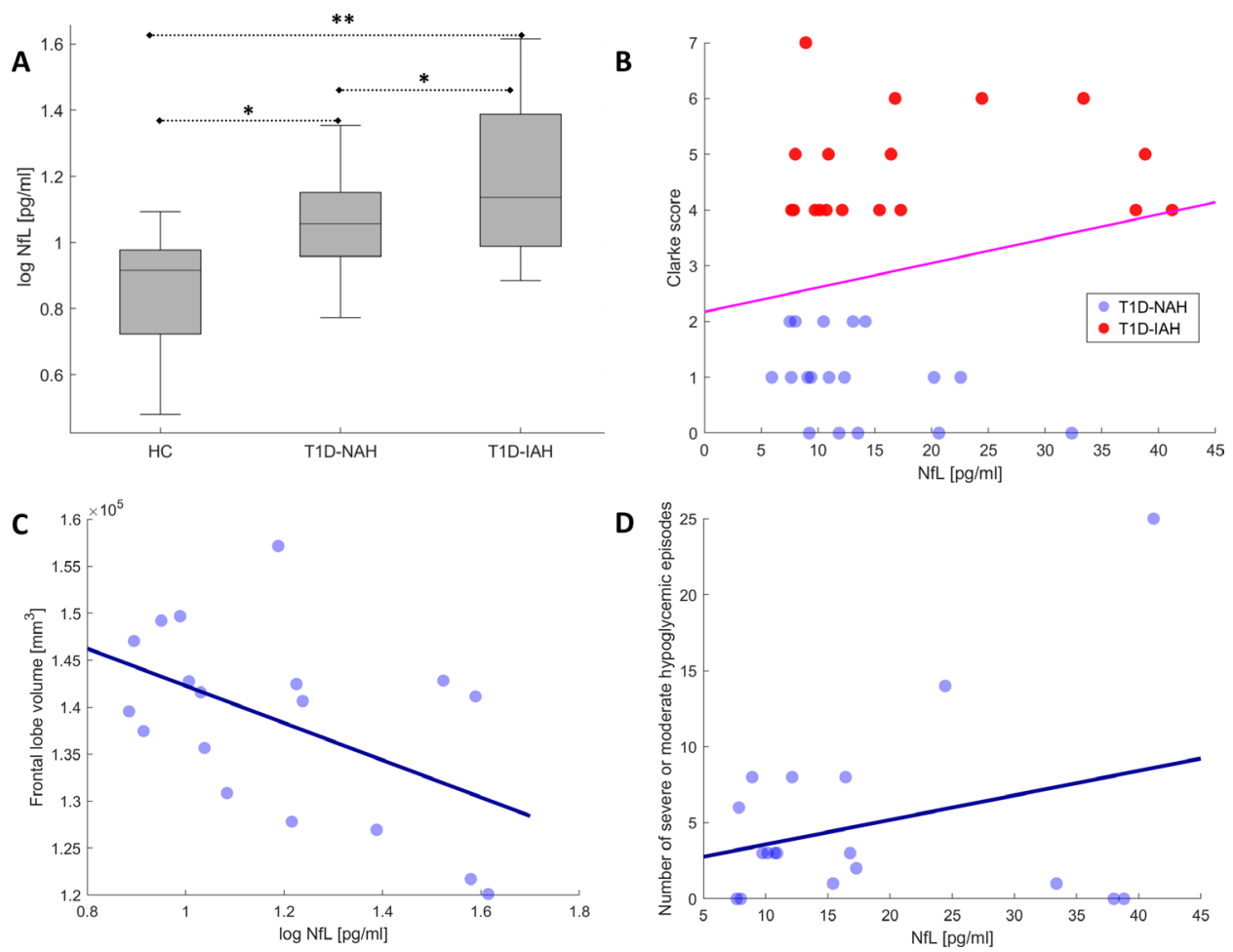

Figure 1 (A) Plasma NfL differences across groups $\left({ }^{*} p<0.05,{ }^{* *} p<0.005\right)$. Scatter plots illustrating a subset of significant associations between NfL levels and (B) Clarke scores in patients with T1D, (C) frontal lobe GMV within the T1D-IAH group, and (D) number of severe or moderate hypoglycemic episodes within the T1D-IAH group. GMV, gray matter volumes; HC, healthy controls; IAH, impaired awareness of hypoglycemia; NAH, normal awareness of hypoglycemia; NfL, neurofilament light chain; T1D, type-1 diabetes.

Patients with T1D-IAH showed reduced cerebral GMV, especially in the frontal lobe. A frontal compromise has been previously described in this population, both in neuroimaging and cognitive studies. ${ }^{510}$ Importantly, in patients with T1D-IAH, increased NfL levels correlated with reduced frontal GMV. This association shows that increased NfL levels reflect cerebral damage in this population, reinforcing its role as a potential biomarker. Moreover, increased NfL levels were also associated with increased IAH severity, as measured by Clarke scores and also by the number of severe or moderate hypoglycemic events. This further emphasizes the clinical significance of increased NfL levels in this population, which probably reflect neural damage due to recurrent hypoglycemia.

The main strength of this study is the inclusion of patients with T1D-IAH and T1D-NAH with similar T1D duration and sociodemographic and cardiovascular profiles. This allowed studying the effect of IAH on NfL levels and cerebral GMV with high specificity. Limitations of this work include the use of a relatively low sample size, some missing data in the control group, and the exploratory character of the correlations between NfL levels and IAH severity and GMV within the T1D-IAH group.

To conclude, we found that plasma NfL levels are increased in patients with T1D-IAH, probably reflecting the presence of neuroaxonal damage with clinical consequences. Overall, our findings motivate further confirmatory studies on the potential role of this biomarker in this population, which could be used to predict IAH or monitor cerebral damage in clinical trials.

Acknowledgements The authors thank Eugenia Mato, CIBER-BBN, Santa Creu i Sant Pau Hospital, for help with the neurofilament processing.

Contributors FS wrote the manuscript and contributed to statistical and image analysis. NS researched data, executed the study, and reviewed the manuscript. SMH reviewed the manuscript. DA, AL, LM, and RPG contributed to NfL processing and reviewed/edited the manuscript. JML reviewed/edited the manuscript. BGA contributed to image analysis and reviewed/edited manuscript. AC researched data and reviewed/edited the manuscript. FS, NS, and AC are the guarantors of this work.

Funding This work was financially supported by a grant from the SPANISH DIABETES SOCIETY.

Competing interests None declared.

Patient consent for publication Not required.

Ethics approval All procedures performed in studies involving human participants were in accordance with the ethical standards of the institutional research committee and with the 1964 Helsinki Declaration and its later amendments or comparable ethical standards. Ethical approval for this study was obtained from the Ethics Committee of the Hospital de la Santa Creu i Sant Pau (http://www.recercasantpau. cat/en/clinical-research/ceic/) on 17 April 2018 at the Hospital de la Santa Creu i Sant Pau, Barcelona, Spain (Code: EC/18/158/5224). All participants provided signed informed consent.

Provenance and peer review Not commissioned; externally peer reviewed.

Data availability statement Data are available on reasonable request.

Open access This is an open access article distributed in accordance with the Creative Commons Attribution Non Commercial (CC BY-NC 4.0) license, which permits others to distribute, remix, adapt, build upon this work non-commercially, and license their derivative works on different terms, provided the original work is 
properly cited, appropriate credit is given, any changes made indicated, and the use is non-commercial. See: http://creativecommons.org/licenses/by-nc/4.0/.

ORCID iD

Ana Chico http://orcid.org/0000-0001-6498-3411

\section{REFERENCES}

1 Geddes J, Schopman JE, Zammitt NN, et al. Prevalence of impaired awareness of hypoglycaemia in adults with type 1 diabetes. Diabet Med 2008;25:501-4.

2 Perros P, Deary IJ, Sellar RJ, et al. Brain abnormalities demonstrated by magnetic resonance imaging in adult IDDM patients with and without a history of recurrent severe hypoglycemia. Diabetes Care 1997:20:1013-8.

3 Dave KR, Tamariz J, Desai KM, et al. Recurrent hypoglycemia exacerbates cerebral ischemic damage in streptozotocin-induced diabetic rats. Stroke 2011;42:1404-11.

4 Reddy M, Jugnee N, El Laboudi A, et al. A randomized controlled pilot study of continuous glucose monitoring and flash glucose monitoring in people with type 1 diabetes and impaired awareness of hypoglycaemia. Diabet Med 2018;35:483-90.

5 Bednarik P, Moheet AA, Grohn H, et al. Type 1 diabetes and impaired awareness of hypoglycemia are associated with reduced brain gray matter volumes. Front Neurosci 2017;11:529.

6 Khalil M, Teunissen CE, Otto M, et al. Neurofilaments as biomarkers in neurological disorders. Nat Rev Neurol 2018;14:577-89.

7 Gaetani L, Blennow K, Calabresi P, et al. Neurofilament light chain as a biomarker in neurological disorders. J Neurol Neurosurg Psychiatry 2019;90:870-81.

8 Clarke WL, Cox DJ, Gonder-Frederick LA, et al. Reduced awareness of hypoglycemia in adults with IDDM. A prospective study of hypoglycemic frequency and associated symptoms. Diabetes Care 1995;18:517-22.

9 Rissin DM, Kan CW, Campbell TG, et al. Single-Molecule enzyme-linked immunosorbent assay detects serum proteins at subfemtomolar concentrations. Nat Biotechnol 2010;28:595-9.

10 Hansen TI, Olsen SE, Haferstrom ECD, et al. Cognitive deficits associated with impaired awareness of hypoglycaemia in type 1 diabetes. Diabetologia 2017;60:971-9. 